\title{
Is serum uric acid a predictive factor for stroke in men with hypertriglyceridemia?
}

\author{
Lai YJ ${ }^{1}$, Hsu CY $2,3,4,5,6,7,8,9,10$ \\ Department of Neurology, China Medical University Hospital, Taichung, Taiwan.4211@mail.pch.org.tw
}

\begin{abstract}
OBJECTIVE: This study investigated the association between serum uric acid (sUA) and stroke risk in men with hypertriglyceridemia.

METHODS: Between 2002 and 2012, male patients with pure hypertriglyceridemia and a triglyceride (TG) level $\geq 150 \mathrm{mg} / \mathrm{dL}$ were enrolled. Eligible patients were categorized into two groups according to their sUA levels ( $\geq$ and $<8 \mathrm{mg} / \mathrm{dL}$ ). Clinical characteristics and comorbidities that are risk factors for stroke were recorded and compared between the groups.

RESULTS: A total of 265 male patients (95 with sUA $\geq 8 \mathrm{mg} / \mathrm{dL}$ and 170 with sUA $<8 \mathrm{mg} / \mathrm{dL}$ ) were enrolled. The incidence of ischemic type of stroke was significantly higher in patients with sUA $\geq 8 \mathrm{mg} / \mathrm{dL}(p=0.038)$, particularly in the age range of 45-65 years. Multivariate Cox proportional analyses confirmed that age $(p=$ 0.003 ) and UA ( $p=0.019$ ) were major predictive factors for stroke free (ischemic type of stroke) survival.

CONCLUSION: Among men with hypertriglyceridemia, the incidence rate of ischemic type of stroke significantly increased with sUA levels $\geq 8 \mathrm{mg} / \mathrm{dL}$, particularly in men aged 45 to 65 years. Hyperuricemia is considered a potential predictive factor for ischemic type of stroke and may indicate the need for preventive management in patients with hypertriglyceridemia (Tab. 3, Fig. 1, Ref. 28). Text in PDF www.elis.sk.

KEY WORDS: hypertriglyceridemia, hyperuricemia, stroke.
\end{abstract}

\section{Introduction}

Stroke is a major global cause of death and disability (1), and thus is a worldwide health issue. Stroke is attributed to ameliorable risk factors (2), hypertension, diabetes, hyperlipidemia, alcohol consumption, smoking, cardiac-related issues and ratio of apolipoprotein B to A1 were found to be strongly associated with an increased risk of ischemic stroke $(3,4)$.

Triglyceride (TG) is an ester that is transported by lipoproteins such as chylomicrons and very low density lipoproteins. According to guidelines published by the American Heart Association, the TG level is considered to be high when it is over $1.7 \mathrm{mmol} / \mathrm{L}$ $(150 \mathrm{mg} / \mathrm{dL})$. Hypertriglyceridemia is one of the components of the metabolic syndrome. A national health and nutrition examination

${ }^{1}$ Department of Neurology, China Medical University Hospital, Taichung, Taiwan, ${ }^{2}$ Department of Family Medicine, Puli Christian Hospital, Puli, Taiwan, ${ }^{3}$ Department of Medical Education and Research, Puli Christian Hospital, Puli, Taiwan, ${ }^{4}$ Department of Optometry, Central Taiwan University of Science and Technology, Taichung, Taiwan, ${ }^{5}$ Center for General Education, National Taichung University of Science and Technology, Taichung, Taiwan, ${ }^{6}$ The General Education Center, Chaoyang University of Technology, Taichung, Taiwan, ${ }^{7}$ Department of General Education, National Chin-Yi University of Technology, Taichung, Taiwan, ${ }^{8} \mathrm{Center}$ for General Education, Feng Chia University, Taichung, Taiwan, ${ }^{9}$ Center for General Education, National Chi Nan University, Puli, Taiwan, and ${ }^{10}$ Rural Generalist Program Japan

Address for correspondence: C-Y Hsu, Tie-Shan Road 1, Puli, Nantou 545, Taiwan.

Phone: 886.492912151, Fax: 886.492916901 survey showed that one third of respondents had hypertriglyceridemia, and that the prevalence of this disease is increasing. A high TG level has been associated with a high risk of cardiovascular disease (CVD), acute pancreatitis, and myocardial infarction $(5,6)$.

Several studies have attempted to identify an association between serum uric acid (sUA) and CVD (7-12). Increased levels of sUA may predict cardiovascular events in a population with a relatively high risk of stroke (8). Increased level of sUA was associated with stroke risk in diabetes patients (7) and was an independent predictor of ischemic stroke in patients who do not take diuretic medication (9). Some studies have reported disappearance of this association after adjustment for various confounding factors $(13,14)$. However, two meta-analyses have found a significant association among hyperuricemia, stroke incidence, and mortality after adjustment for known cardiovascular risk factors (10, 11). Thus, an association among sUA, hypertriglyceridemia, and stroke may exist. This study investigated the possible association between sUA and stroke risk in men with hypertriglyceridemia.

\section{Methods}

Between 2002 and 2012, male patients with pure hypertriglyceridemia and a TG level $\geq 150 \mathrm{mg} / \mathrm{dL}$ (without previous TG level $\geq 150 \mathrm{mg} / \mathrm{dL}$ for at least a one-year tracking time) were enrolled in this study. Because sex differences between sUA and several diseases have been described in previous studies $(15,16)$, only male patients were recruited. Patients were excluded if they had a medical history of stroke (a), total follow-up period of less than 
1 year (b), showed a high average cholesterol level ( $\geq 200 \mathrm{mg} / \mathrm{dL})$ (c), and were under hemodialysis within one year before or after the date when participants satisfied the criteria (d). This was to eliminate the effect of high serum cholesterol, and to ensure that the effect of pure hypertriglyceridemia is observed. In addition, we excluded patients with hemodialysis. This is because for those on dialysis, sUA is a factor that reflects a patient's nutritional status, while low sUA level is associated with high mortality $(17,18)$.

No precise cut-off value of sUA was recommended for initial urate-lowering therapy (ULT) in managing asymptomatic hyperuricemia, and gout attack in hyperuricemic individuals with sUA levels between 7 and $8 \mathrm{mg} / \mathrm{dL}$ was low (19). We used $8 \mathrm{mg} / \mathrm{dL}$ as the cut-off point. Eligible patients were categorized into two groups according to their sUA levels ( $\geq$ and $<8 \mathrm{mg} / \mathrm{dL}$ ). Clinical characteristics (age at diagnosis and race/ethnicity), and comorbidities that are risk factors for stroke (hypertension, diabetes, obesity, and atrial fibrillation; 20) were recorded and compared between the groups. No age restrictions were imposed. To identify age differences in patients based on sUA levels and stroke in men with hypertriglyceridemia, we divided patients into three age groups: $<45,45-65$, and $>65$ years.

Study outcomes were stroke events of either ischemic or hemorrhagic types, in which there was at least one hospital discharge diagnosis for stroke, or at least two outpatient clinical diagnoses with detailed neuronal examinations documented in medical records with computed tomography/ magnetic resonance imaging confirmed.

Chi-square and independent $\mathrm{t}$ tests were used to compare the patients' characteristics, comorbidities, and events of stroke in the follow-up period. Stroke free survival rate was evaluated by plotting Kaplan-Meier curves. Univariable and multivariable Cox regression analyses were used to assess the hazard ratio of each possible confounding factor and its impact on stroke risk and to identify the prognostic factors of stroke events. A p value $<0.05$ was considered significant.

Tab. 1. The differences between high and low levels of uric acid in men with pure hypertriglyceridemia.

\begin{tabular}{lccc}
\hline & $\begin{array}{c}\mathrm{sUA} \geq 8 \mathrm{mg} / \mathrm{dL} \\
\mathrm{N}=95\end{array}$ & $\begin{array}{c}\mathrm{sUA}<8 \mathrm{mg} / \mathrm{dL} \\
\mathrm{N}=170\end{array}$ & $\mathrm{p}$ \\
\hline Age at diagnosis & $46.9 \pm 13.7$ & $48.8 \pm 13.0$ & 0.252 \\
\hline Mean follow time (year) & $10.8 \pm 4.4$ & $11.3 \pm 3.7$ & 0.308 \\
\hline Mean triglyceride & $365.0 \pm 192.0$ & $358.4 \pm 239.7$ & 0.819 \\
\hline Mean uric acid & $9.1 \pm 1.0$ & $6.4 \pm 1.1$ & $<0.001$ \\
\hline Race & & & \\
$\quad$ Aborigines & $20(21.1 \%)$ & $28(16.5 \%)$ & 0.353 \\
$\quad$ Non-aborigines & $75(78.9 \%)$ & $142(83.5 \%)$ & \\
\hline Comorbidities & & & \\
$\quad$ Hypertension & $41(43.2 \%)$ & $81(47.7 \%)$ & 0.427 \\
Diabetes & $21(22.1 \%)$ & $59(34.7 \%)$ & 0.040 \\
Obesity & $17(17.9 \%)$ & $25(14.7 \%)$ & 0.582 \\
$\quad$ Atrial fibrillation & $2(2.1 \%)$ & $2(1.2 \%)$ & 0.552 \\
\hline Stroke & $14(14.8 \%)$ & $15(8.8 \%)$ & 0.139 \\
$\quad$ Ischemic & $11(11.6 \%)$ & $8(4.7 \%)$ & 0.038 \\
Hemorrhagic & $3(3.2 \%)$ & $7(4.1 \%)$ & 0.694 \\
\hline
\end{tabular}

Tab. 2. The incidence of ischemic type of stroke by different age groups.

\begin{tabular}{lcccc}
\hline Age & $\begin{array}{c}\text { Uric acid } \\
\text { level }\end{array}$ & $\begin{array}{c}\text { Number } \\
\text { of patients }\end{array}$ & $\begin{array}{c}\text { Number of } \\
\text { ischemic strokes }\end{array}$ & $\mathrm{p}$ \\
\hline$<45$ & $\geq 8$ & 45 & $3(6.7 \%)$ & 0.153 \\
\hline \multirow{2}{*}{$45-65$} & $<8$ & 66 & $1(1.5 \%)$ & \\
\hline$>65$ & $<8$ & 41 & $6(14.6 \%)$ & 0.004 \\
\hline & $<8$ & 76 & $1(1.3 \%)$ & 0.960 \\
\hline
\end{tabular}

\section{Results}

A total of 265 male patients ( 95 with sUA levels $\geq 8 \mathrm{mg} / \mathrm{dL}$ and 170 with sUA levels $<8 \mathrm{mg} / \mathrm{dL}$ ) were enrolled. The mean age was 46.9 years for patients with sUA levels $\geq 8 \mathrm{mg} / \mathrm{dL}$ and 48.8 years for those with $\mathrm{sUA}<8 \mathrm{mg} / \mathrm{dL}(\mathrm{p}=0.252)$. The mean follow-up period was 10.8 years for patients with sUA levels $\geq 8$ $\mathrm{mg} / \mathrm{dL}$ and 11.3 years for those with sUA levels $<8 \mathrm{mg} / \mathrm{dL}$ ( $\mathrm{p}=$ 0.308). Baseline characteristics between the groups were not significantly different except for diabetes (Tab. 1). The incidence of ischemic type of stroke was significantly higher in patients with sUA levels $\geq 8 \mathrm{mg} / \mathrm{dL}$ (11.6 \% vs $4.7 \%$; $\mathrm{p}=0.038$ ). However, no difference in hemorrhagic type of stroke was found between the groups (Tab. 1).

To examine the effect of sUA on ischemic type of stroke in different age groups, we divided the patients into three age groups: $<45,45-65$, and $>65$ years. In the age group of $45-65$ years, the incidence of ischemic type of stroke was significantly higher in patients with sUA levels $\geq 8 \mathrm{mg} / \mathrm{dL}$ than in those with sUA levels $<8 \mathrm{mg} / \mathrm{dL}(14.6 \%$ vs $1.3 \%$; $=0.004)$ (Tab. 2$)$.

The 10-year stroke free (ischemic type of stroke) survival rate was $91.2 \%$ for the patients with sUA levels $\geq 8 \mathrm{mg} / \mathrm{dL}$ and 96.8 $\%$ for those with sUA levels $<8 \mathrm{mg} / \mathrm{dL}$. Kaplan-Meier curves showed a significant difference $(\mathrm{p}=0.034)$ (Fig. 1). The results of the univariate Cox proportional analyses showed that age and sUA were significantly associated with stroke free (ischemic type of stroke) survival. The findings of multivariate Cox proportional analyses confirmed that age and sUA were significant predictive factors for stroke free (ischemic type of stroke) survival $(p=0.003$ for age and 0.019 for sUA) (Tab. 3).

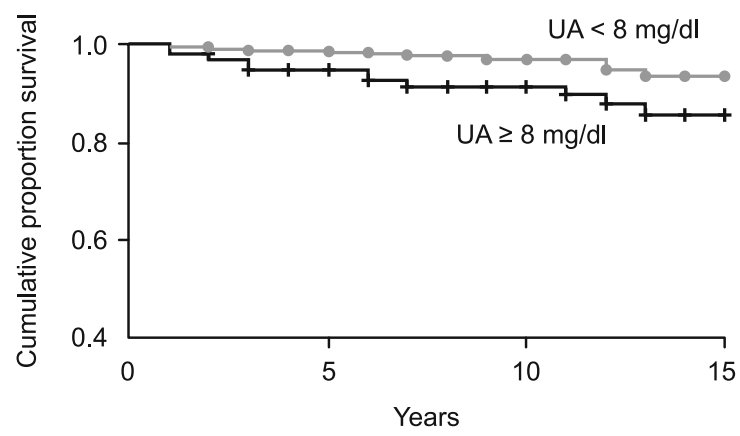

Fig. 1. The stroke free (ischemic type of stroke) survival rate between sUA $\geq 8$ and $<8 \mathrm{mg} / \mathrm{dl}$ by Kaplan-Meier method. 
Tab. 3. The uni- and multivariate Cox analyses for stroke free (ischemic type of stroke) survival.

\begin{tabular}{|c|c|c|c|c|c|c|}
\hline \multirow{2}{*}{ Covariates } & \multicolumn{3}{|c|}{ Univariate analysis } & \multicolumn{3}{|c|}{ Multivariate analysis } \\
\hline & HR & $95 \% \mathrm{CI}$ & $\mathrm{p}$ & HR & $95 \%$ CI & $\mathrm{p}$ \\
\hline Age & 1.054 & $1.019-1.090$ & 0.002 & 1.055 & $1.018-1.093$ & 0.003 \\
\hline Hypertension & 1.067 & $0.434-2.627$ & 0.887 & 1.000 & $0.386-2.588$ & 0.999 \\
\hline Diabetes & 2.285 & $0.928-5.625$ & 0.072 & 2.109 & $0.781-5.693$ & 0.141 \\
\hline Atrial fibrillation & 4.994 & $0.660-37.816$ & 0.119 & 1.915 & $0.206-17.765$ & 0.568 \\
\hline Obesity & 1.441 & $0.478-4.344$ & 0.516 & 1.506 & $0.456-4.966$ & 0.502 \\
\hline Uric acid & 2.573 & $1.035-6.397$ & 0.042 & 3.120 & $1.203-8.092$ & 0.019 \\
\hline
\end{tabular}

\section{Discussion}

The atherogenic effect of lipoproteins on patients with hypertriglyceridemia has been previously confirmed (21). In the present study, we examined the relationship between sUA and stroke in men with hypertriglyceridemia. We found that the incidence rate of ischemic type of stroke increased significantly in patients with sUA $\geq 8 \mathrm{mg} / \mathrm{dL}$, particularly in men aged 45 to 65 years. In addition, we found that age and sUA were significant predictive factors for ischemic type of stroke.

The role of sUA in humans has been previously discussed. It is associated with increased numbers of indicators of inflammation such as interleukin-6, interleukin-18, C-reactive protein, and tumor necrosis factor-alpha (22). In addition to inflammation status, sUA is associated with carotid intima-media thickness, which might lead to atherosclerosis (23). Atherogenesis and thrombogenesis are believed to play a major role in the development of coronary artery disease, and most strokes are caused by occlusion of the bloodstream (24). A high sUA level is considered to increase the stroke rate through atherogenesis. In addition, sUA impairs endothelial function by reducing nitric oxide synthase. Thus, hyperuricemia can be a risk factor for endothelial dysfunction $(25,26)$. Atherogenesis or endothelial dysfunction through the sUA effect increases the incidence of stroke. Our study demonstrated that a high sUA level is a significant predictive factor for ischemic type of stroke.

Age is an irreversible risk factor for ischemic stroke (3). Although Wen et al. found that the mean sUA level in men decreased slightly as age increased (27), Singh and Yu demonstrated that age is a significant factor for incidence of stroke in old patients who take allopurinol (28). Various risk factors including age, family history, hypertension, diabetes, hyperlipidemia, alcohol consumption, smoking, and cardiac-related issues (atrial fibrillation), are associated with an increased risk of ischemic stroke (3). Our study was limited to male patients, and except for age, we did not find any associations between comorbidities and ischemic stroke. One possible explanation is that identification of these factors relies on the coding from the International Classification of Diseases, Ninth Revision, Clinical Modification (ICD-9-CM). There may be a proportion of patients under treatment for certain diseases in local clinics who have not informed doctors at our hospital. Another explanation is that this was a population-based retrospective study with a distinct population component (nearly $19 \%$ of patients were aborigines) and life habits. Thus, the effect scale of each factor to ischemic stroke might be different from that in the general population.
This retrospective study identified an association between sUA and stroke risk in men with hypertriglyceridemia. However, this study has several limitations. Firstly, because sUA is not a routine item in a physical examination, some patients have their first sUA checked only after a gout attack. This may have produced a bias in selecting participants; thus, the lack of sUA data resulting from this may have also limited our study. Secondly, because it was a retrospective study, data related to the life-styles of participants, such as the amount of cigarette smoking and alcohol consumption, were not available in our study based solely on reviews of patient medical records. Thus, we were unable to consider these factors. Thirdly, patients may have been under ULT after the diagnosis of gout. We did not record the fact whether participants were taking medication with ULT, which may have also influenced the results. Fourthly, this study was based on a database from a single hospital, and our study participants mainly reside in a rural area of central Taiwan. The lifestyle preferences and ethnic makeup of these participants may have influenced the results. Nevertheless, despite these limitations, ours is the first study to evaluate the association between sUA and stroke risk in a specific population of men with hypertriglyceridemia.

\section{Conclusion}

Among men with hypertriglyceridemia, the incidence rate of ischemic type of stroke significantly increased with sUA levels $\geq$ $8 \mathrm{mg} / \mathrm{dL}$, particularly in the age group of 45-65 years. Hyperuricemia is considered a potential predictive factor for ischemic type of stroke and may require preventive management in patients with hypertriglyceridemia.

\section{References}

1. GBD 2013 Mortality and Causes of Death Collaborators. Global, regional, and national age-sex specific all-cause and cause-specific mortality for 240 causes of death, 1990-2013: a systematic analysis for the Global Burden of Disease Study 2013. Lancet 2015; 385 (9963): 117-171.

2. Donnan GA, Fisher M, Macleod M, Davis SM. Stroke. Lancet 2008; 371 (9624): 1612-1623.

3. O'Donnell MJ, Xavier D, Liu L, Zhang H, Chin SL, Rao-Melacini $\mathbf{P}$ et al. Risk factors for ischaemic and intracerebral haemorrhagic stroke in 22 countries (the INTERSTROKE study): a case-control study. Lancet 2010; 376 (9735): 112-123.

4. Sarbazi E, Sarbakhsh P, Savadi Oskooei D, Yazdchi M, GhaffariFam S, Shamshirgaran SM. Factors related to 6-month mortality after the first-ever stroke. J Educ Health Promot 2018; 7: 113. 
5. LeBlanc S, Coulombe F, Bertrand OF, Bibeau K, Pibarot P, Marette A et al. Hypertriglyceridemic waist: a simple marker of high-risk atherosclerosis features associated with excess visceral adiposity/ectopic fat. J Am Heart Assoc 2018; 7 (8): pii e008139

6. Pedersen SB, Langsted A, Nordestgaard BG. Nonfasting mild-tomoderate hypertriglyceridemia and risk of acute pancreatitis. JAMA Intern Med 2016; 176 (12): 1834-1842.

7. Lehto S, Niskanen L, Rönnemaa T, Laakso M. Serum uric acid is a strong predictor of stroke in patients with non-insulin-dependent diabetes mellitus. Stroke 1998; 29 (3): 635-639.

8. Chien KL, Hsu HC, Sung FC, Su TC, Chen MF, Lee YT. Hyperuricemia as a risk factor on cardiovascular events in Taiwan: the ChinShan community cardiovascular cohort study. Atherosclerosis 2005; 183 (1): 147-155.

9. Hozawa A, Folsom AR, Ibrahim H, Javier Nieto F, Rosamond WD, Shahar E. Serum uric acid and risk of ischemic stroke: the ARIC Study. Atherosclerosis 2006; 187 (2): 401-407.

10. Kim SY, Guevara JP, Kim KM, Choi HK, Heitjan DF, Albert DA. Hyperuricemia and risk of stroke: a systematic review and meta-analysis. Arthritis Rheum 2009; 61 (7): 885-892.

11. Li M, Hou W, Zhang X, Hu L, Tang Z. Hyperuricemia and risk of stroke: a systematic review and meta-analysis of prospective studies. Atherosclerosis 2014; 232 (2): 265-270.

12. Chang CC, Wu CH, Liu LK, Chou RH, Kuo CS, Huang PH et al. Association between serum uric acid and cardiovascular risk in nonhypertensive and nondiabetic individuals: the Taiwan I-Lan longitudinal aging study. Sci Rep 2018; 8 (1): 5234.

13. Culleton BF, Larson MG, Kannel WB, Levy D. Serum uric acid and risk for cardiovascular disease and death: the Framingham heart study. Ann Intern Med 1999; 131 (1): 7-13.

14. Moriarity JT, Folsom AR, Iribarren C, Nieto FJ, Rosamond WD. Serum uric acid and risk of coronary heart disease: atherosclerosis risk in communities (ARIC) study. Ann Epidemiol 2000; 10 (3): 136-143.

15. Liu J, Zhao Z, Mu Y, Zou X, Zou D, Zhang J et al. Gender differences in the association between serum uric acid and prediabetes: a sixyear longitudinal cohort study. Int J Environ Res Public Health 2018; 5 (7): pii: E1560.

16. Zheng X, Wei Q, Long J, Gong L, Chen H, Luo R, et al. Genderspecific association of serum uric acid levels and cardio-ankle vascular index in Chinese adults. Lipids Health Dis 2018; 17 (1): 80.
17. Beberashvili I, Sinuani I, Azar A, Shapiro G, Feldman L, Stav K et al. Serum uric acid as a clinically useful nutritional marker and predictor of outcome in maintenance hemodialysis patients. Nutrition 2015; 31 (1): 138-147.

18. Bae E, Cho HJ, Shin N, Kim SM, Yang SH, Lim DK et al. Lower serum uric acid level predicts mortality in dialysis patients. Medicine (Baltimore) 2016; 95 (24): e3701.

19. Choi H K, Mount DB, Reginato AM, American College of Physicians, American Physiological Society. Pathogenesis of Gout. Ann Intern Med 2005; 143 (7): 499-516.

20. Chen Y, Xia Y, Han X, Yang Y, Yin X, Qiu J et al. Association between serum uric acid and atrial fibrillation: a cross-sectional communitybased study in China. BMJ Open 2017; 7 (12): e019037.

21. Gotto AM Jr. Interrelationship of triglycerides with lipoproteins and high-density lipoproteins. Am J Cardiol 1990; 66 (6): 20A-23A.

22. Lyngdoh T, Marques-Vidal P, Paccaud F, Preisig M, Waeber G, Bochud $M$ et al. Elevated serum uric acid is associated with high circulating inflammatory cytokines in the population-based Colaus study. PLoS ONE 2011; 6 (5): e19901.

23. Montalcini T, Gorgone G, Gazzaruso C, Sesti G, Perticone F, Pujia A. Relation between serum uric acid and carotid intima-media thickness in healthy postmenopausal women. Intern Emerg Med 2007; 2 (1): 19-23.

24. Humphries SE, Morgan L. Genetic risk factors for stroke and carotid atherosclerosis: insights into pathophysiology from candidate gene approaches. Lancet Neurol 2004; 3 (4): 227-235.

25. Hong Q, Qi K, Fen Z, Huang Z, Cui S, Wang L et al. Hyperuricemia induced endothelial dysfunction via mitochondrial $\mathrm{Na}+/ \mathrm{Ca} 2+$ exchanger-mediated mitochondrial calcium overload. Cell Calcium 2012; 51 (5): 402-410.

26. Steinberg HO, Brechtel G, Johnson A, Fineberg N, Baron AD. Insulin-mediated skeletal muscle vasodilation is nitric oxide dependent. A novel action of insulin to increase nitric oxide release. J Clin Invest 1994; 94 (3): 1172-1179.

27. Wen CP, David Cheng TY, Chan HT, Tsai MK, Chung WS, Tsai SP et al. Is high serum uric acid a risk marker or a target for treatment? Examination of its independent effect in a large cohort with low cardiovascular risk. Am J Kidney Dis 2010; 56 (2): 273-288.

28. Singh JA, Yu SH. Allopurinol and the risk of stroke in older adults receiving medicare. BMC Neurol 2016; 16 (1): 164.

Received December 14, 2018. Accepted January 25, 2019. 